2. Severe hypoxaemia is commonly defined as $\mathrm{P}_{\mathrm{a}} \mathrm{O}_{2}<60 \mathrm{mmHg}$. This correlates to a $\mathrm{SpO}_{2}$ reading of what?
(A) $87 \%$
(B) $90 \%$
(C) $93 \%$
(D) $97 \%$

3. Which of the following is NOT a potential cause of hypoxaemia (low blood oxygen)?
(A) Ventilation-perfusion (V/Q)
(B) Low inspired oxygen levels $\left(\mathrm{FiO}_{2}\right)$
(C) Hypoventilation
(D) Hypocapnia

\section{CPCR in the anaesthetized patient}

\section{Joanne Michou}

Survival rates following cardiopulmonary arrest (CPA) to discharge are relatively low in veterinary medicine, estimated at $3-6 \%$ in dogs and cats, compared to $20 \%$ in humans that experience in-hospital CPA. However, with appropriate monitoring, understanding potential complications for each patient condition, procedure or anaesthetic plan, being fully prepared and using safety checklists, it is feasible to reduce this number significantly by reducing error and detecting abnormalities earlier. Resuscitation attempts that are organized, cohesive and led by well functioning knowledgeable teams adhering to evidence-based cardiopumonary resuscitation (CPR) guidelines, RECOVER 2012 (The Reassessment Campaign On Veterinary Resuscitation) should improve survival from CPA.

\section{RECOGNITION OF CARDIOPULMONARY ARREST}

In anaesthetized patients, CPA may be avoided if monitoring is effective, allowing cardiorespiratory alterations to be picked up earlier, such as sudden change in heart rate, change in electrocariogram (ECG) complexes or decrease in end-tidal carbon dioxide $\left(\mathrm{EtCO}_{2}\right)$. There is high-level evidence that anaesthesia-related CPAs are associated with increased survival compared to CPAs from other causes.

\section{WHEN TO START CPR}

Assessment for CPA should be rapid and last no more than 15 seconds. The deleterious delay in starting BLS is significant. With serious adverse effects occurring in less than $2 \%$ of human patients not in CPA that receive chest compressions, and the potential benefits of early chest compressions in patients in CPA, chest compressions should be provided to dogs and cats as soon as possible if CPA cannot be definitively ruled out.

\section{BASIC LIFE SUPPORT}

BLS consists of CAB: Circulation, Airway, Breathing. The guidelines stress the importance of early delivery of high-quality chest compressions with minimal interruption. In addition, it is likely that early intubation and ventilation in veterinary CPR is highly valuable. All anaesthetized patients should have an endotracheal tube in place with a breathing system attached, providing the ability to ventilate the patient immediately.

Depending on the surgical site, open-chest CPR may be appropriate.

\section{ADVANCED LIFE SUPPORT}

Monitoring and vascular access should be in place for any anaesthetized patient, but available monitoring is widely variable between veterinary practices.

- $\mathrm{EtCO}_{2}$ is highly valuable to identify return of spontaneous circulation (ROSC) and maintaining $\mathrm{EtCO}_{2}>15 \mathrm{mmHg}$ indicates effective compressions

- ECG to identify rhythm and decide on drug therapy (adrenaline/epinephrine) or defibrillation

- Pulse oximeter or a Doppler probe on the cornea can detect pulsatile blood flow.

Administer reversals, target treatment:

- Turn off anaesthetic, empty breathing system

- Reverse/treat cause if known

- For animals with increase vagal tone the use of atropine is reasonable

- Assess for other causes and treat: 4Hs (hypoxia, hypovolaemia/hypotension, hyperkalaemia, hypothermia) and 4Ts (thrombosis, tension pneumothorax, tamponade-cardiac, toxins).

\section{OPTIONS AND AIM OF ELECTROCARDIOGRAM}

During each 2 minutes of BLS inter-cycles, assess if the rhythm is shockable (ventricular tachycardia, ventricular fibrillation), non-shockable (asystole, pulseless electrical activity) or you have ROSC:

- What is the arrest rhythm?

- Is there a pulse?

\section{POST-ARREST CARE}

Post-cardiac arrest monitoring should be sufficient to detect impending recurrence of CPA. Do not remove monitoring! Goal-directed therapy must be individually tailored to each patient. 


\section{Anaesthesia}

\section{KEY LEARNING OBJECTIVES}

- Appreciate the importance of monitoring and team communication

- Learn what basic life support (BLS) is and importance of chest compressions

- Be prepared to implement and adapt RECOVER in anaesthetized patients

\section{MULTIPLE CHOICE QUESTIONS}

1. Which of the following is correct about anaesthesia-related CPAs compared to CPAs from other causes?

(A) Not likely to survive at all

(B) Associated with decreased survival
(C) Associated with increased survival

(D) None of the above

2. How long is each BLS cycle?
(A) 30 seconds
(B) 1 minute
(C) 2 minutes
(D) 4 minutes

3. Which method of monitoring is very useful to identify ROSC?
(A) Pulse oximetry
(B) Capnography
(C) Electrocardiogram
(D) Non-invasive blood pressure 